\title{
HOMOLOGY OPERATIONS AND POWER SERIES
}

\author{
by RICHARD STEINER
}

(Received 2 February, 1982)

1. Introduction. Bullett and Macdonald [1] have used power series to simplify the statement and proof of the Adem relations for Steenrod cohomology operations. In this paper I give a similar treatment of May's generalized Adem relations [4, §4] and of the Nishida relations $([6],[2, \mathbf{I} .1 .1(9)],[5,3.1(7)])$. Both sets of relations apply to DyerLashof operations in $E_{\infty}$ spaces such as infinite loop spaces ([3], [2, I.1]) and in $H_{\infty}$ ring spectra $([5, \S 3])$.

The results on the Adem relations are very similar to those of [1], and the proof mostly comes from [4]. The surprising result of this paper is that the Adem and Nishida relations can be expressed by virtually the same formulae $((2 a)$ and $(5 a),(2 c)$ and $(5 b)$ below). My proof of the Nishida relations is designed to explain this, and is a bit simpler than the original proof.

Throughout the paper we work modulo $p$, where $p$ is a prime number.

2. Statement of the Adem relations. Recall that in [4] May constructs operations in the homology of certain modulo $p$ chain complexes. There are operations $P_{i}$ for all $p$ and $\beta P_{i}$ for odd $p$, where $i$ is any integer. The degree of $P_{i}$ is $i$ if $p=2$ or $2 i(p-1)$ if $p$ is odd, and the degree of $\beta P_{i}$ is $2 i(p-1)-1$. The Dyer-Lashof operations denoted $Q^{i}$ and $\beta Q^{i}$ are special cases of the $P_{i}$ and $\beta P_{i}$, and the Steenrod cohomology operations denoted $P^{i}$ (or $S q^{i}$ if $p=2$ ) and $\beta P^{i}$ are special cases of the $P_{-i}$ and $\beta P_{-i}$ (a $q$ th cohomology group being regarded as a $(-q)$ th homology group).

We consider complexes for which all the operations are defined and the Adem relations hold: Adem objects of $\mathscr{C}(p, \infty)$ in the terminology of [4]. Let $u$ and $v$ be indeterminates commuting with each other and with anything else that occurs, and put

$$
t=v\left(1-u^{-1} v\right)^{p-1}, \quad s=u\left(1-v^{-1} u\right)^{p-1}=u^{p} v^{-p} t=u^{p} v^{-(p-1)}\left(1-u^{-1} v\right)^{p-1} .
$$

For any $z$, write

$$
P(z)=\sum_{i} z^{i} P_{i}, \quad \beta P(z)=\sum_{i} z^{i} \beta P_{i} ;
$$

(all summations are over the integers, and terms usually regarded as undefined are to be taken as zero). The Adem relations can then be written: for all $p$,

and for odd $p$,

$$
P(u) P(t)=P(v) P(s),
$$

$$
\begin{aligned}
\beta P(u) P(t) & =\beta P(v) P(s), \\
P(u) \beta P(t) & =\left(1-u^{-1} v\right) \beta P(v) P(s)+u^{-1} v P(v) \beta P(s), \\
\beta P(u) \beta P(t) & =u^{-1} v \beta P(v) \beta P(s) .
\end{aligned}
$$

The usual Adem relations are derived in Section 5, and $(2 a)-(2 d)$ are proved in Section 7.

Glasgow Math. J. 24 (1983) 161-168. 
REMARK 1 . In the applications mentioned above, $\beta P_{i}$ is the composite of $P_{i}$ with a Bockstein $\beta$ satisfying $\beta^{2}=0$, so that $(2 a)$ implies $(2 b)$ and $(2 c)$ implies $(2 d)$. But this is not true in general (despite the notation), which is why $(2 b)$ and $(2 d)$ are stated separately.

Remark 2. To get formulae for cohomology operations, write $P^{*}(z)=\sum_{i} z^{i} P^{i}$, etc. $\left(P^{*}(z)\right.$ is denoted $P(z)$ in $\left.[\mathbf{1}]\right)$. Since $P^{\mathrm{i}}$ is an example of $P_{-i}$, the relations (2a)-(2d) imply

$$
P^{*}\left(u^{-1}\right) P^{*}\left(t^{-1}\right)=P^{*}\left(v^{-1}\right) P^{*}\left(s^{-1}\right),
$$

etc. These relations are not the same as in [1], but are equivalent, as is shown in $[\mathbf{1}, \S 4]$ for $p=2$.

3. Statement of the Nishida relations. The Nishida relations apply to the homology of an $E_{\infty}$ space or $H_{\infty}$ ring spectrum. They link the Dyer-Lashof operations $Q^{i}$, the Bockstein $\beta$, and the duals $P_{*}^{\mathrm{i}}$ of the Steenrod cohomology operations. With $u, v, s$ and $t$ as in (1) and with the obvious meanings for $Q(z)$ and $P_{*}(z)$ they say: for all $p$,

$$
P_{*}\left(u^{-1}\right) Q(v)=Q(t) P_{*}\left(s^{-1}\right)
$$

and for odd $p$,

$$
P_{*}\left(u^{-1}\right) \beta Q(v)=\left(1-u^{-1} v\right)^{-1}\left[\beta Q(t) P_{*}\left(s^{-1}\right)-u^{-1} v Q(t) P_{*}\left(s^{-1}\right) \beta\right] .
$$

These can be rewritten to look like the Adem relations $(2 a)$ and $(2 c)$ if one uses the conjugate cohomology operations $c P^{i}$ defined by

$$
\begin{aligned}
& \sum_{i+j=k}\left(c P^{i}\right) P^{j}=1 \text { if } k=0, \\
& 0 \text { if } k \neq 0 .
\end{aligned}
$$

This definition can be written $c P^{*}(z) P^{*}(z)=1$ with the obvious notation, and dualization yields

$$
c P_{*}(z)=P_{*}(z)^{-1} .
$$

So $(3 a)$ is equivalent to: for all $p$,

$$
c P_{*}\left(u^{-1}\right) Q(t)=Q(v) c P_{*}\left(s^{-1}\right) .
$$

Substitution of $(3 a)$ in $(3 b)$ yields

$$
P_{*}\left(u^{-1}\right) \beta Q(v)=\left(1-u^{-1} v\right)^{-1}\left[\beta Q(t) P_{*}\left(s^{-1}\right)-u^{-1} v P_{*}\left(u^{-1}\right) Q(v) \beta\right]
$$

which by (4) is similarly equivalent to: for odd $p$,

$$
c P_{*}\left(u^{-1}\right) \beta Q(t)=\left(1-u^{-1} v\right) \beta Q(v) c P_{*}\left(s^{-1}\right)+u^{-1} v Q(v) \beta c P_{*}\left(s^{-1}\right) .
$$

The usual Nishida relations will be derived from $(3 a)-(3 b)$ in Section 6 and $(5 a)-(5 b)$ will be proved from $(2 a)$ and $(2 c)$ in Section 8 . 
4. The Cartan formulae. For completeness we note that the Cartan formulae

$$
P_{k}(x \otimes y)=\sum_{i+j=k} P_{i} x \otimes P_{i} y
$$

$[4,2.7]$ are obviously equivalent to

$$
P(z)(x \otimes y)=P(z) x \otimes P(z) y,
$$

and similarly for other Cartan formulae. One, which will be used in Section 8 , is

$$
c P_{*}(z)(x \otimes y)=c P_{*}(z) x \otimes c P_{*}(z) y \text {. }
$$

5. Derivation of the usual Adem relations. The usual Adem relations $[4,4.7]$ are: for all $p$,

$$
P_{a} P_{b}=\sum_{i}(-1)^{a+i}\left(\begin{array}{c}
(i-b)(p-1)-1 \\
i p-a
\end{array}\right) P_{a+b-i} P_{i},
$$

and for odd $p$,

$$
\begin{aligned}
\beta P_{a} P_{b} & =\sum_{i}(-1)^{a+i}\left(\begin{array}{c}
(i-b)(p-1)-1 \\
i p-a
\end{array}\right) \beta P_{a+b-i} P_{i} \\
P_{a} \beta P_{b} & =\sum_{i}(-1)^{a+i}\left(\begin{array}{c}
(i-b)(p-1) \\
i p-a
\end{array}\right) \beta P_{a+b-i} P_{i}-\sum_{i}(-1)^{a+i}\left(\begin{array}{c}
(i-b)(p-1)-1 \\
i p-a-1
\end{array}\right) P_{a+b-i} \beta P_{i}, \\
\beta P_{a} \beta P_{b} & =-\sum_{i}(-1)^{a+i}\left(\begin{array}{c}
(i-b)(p-1)-1 \\
i p-a-1
\end{array}\right) \beta P_{a+b-i} \beta P_{i} .
\end{aligned}
$$

REMARK. The binomial coefficients are defined by

$$
\left(\begin{array}{l}
n \\
k
\end{array}\right)=\left\{\begin{array}{lll}
\frac{n(n-1) \ldots(n-k+1)}{k !} & \text { if } & k>0 \\
1 & \text { if } & k=0 \\
0 & \text { if } & k<0
\end{array}\right.
$$

regardless of the sign of $n$. With these conventions, the relations are true for all $a$ and $b$.

The relations are derived from $(2 a)-(2 d)$ respectively by the method of $[1, \S 3]$ : express the right hand sides of $(2 a)-(2 d)$ as power series in $u, v, t$ by putting $s=u^{\mathrm{p}} v^{-\mathrm{p} t}$ (see (1)), then express them as power series in $u$ and $t$ by putting

$$
v^{r}=\sum_{k}(-1)^{k-r}\left(\begin{array}{c}
-k(p-1)-1 \\
k-r
\end{array}\right) u^{r-k} t^{k}
$$

(which is true modulo $p$ and will be proved below), and finally equate the coefficients of $u^{a} t^{b}$.

Proof of (7). Put $V=u^{-1} v, T=u^{-1} t$; then (7) is equivalent to

$$
V^{r}=\sum_{k}(-1)^{k-r}\left(\begin{array}{c}
-k(p-1)-1 \\
k-r
\end{array}\right) T^{k}
$$


But (1) gives

which modulo $p$ gives

$$
T=V(1-V)^{p-1}
$$

so

$$
d T=(1-V)^{\mathrm{p}-2} d V
$$

$$
\begin{aligned}
V^{r}=\sum_{k}\left(\underset{T=0}{\operatorname{res}} V^{r} T^{-k-1} d T\right) T^{k} & =\sum_{k}\left(\underset{V=0}{\operatorname{res}} V^{r-k-1}(1-V)^{-k(p-1)-1} d V\right) T^{k} \\
& =\sum_{k}(-1)^{k-r}\left(\begin{array}{c}
-k(p-1)-1 \\
k-r
\end{array}\right) T^{k},
\end{aligned}
$$

as required.

6. Derivation of the usual Nishida relations. The usual Nishida relations ([6], [2, I.1.1(9)], [5, 3.1(7)]) are: for all $p$,

$$
P_{*}^{m} Q^{n}=\sum_{i}(-1)^{m+i}\left(\begin{array}{c}
(n-m)(p-1) \\
m-i p
\end{array}\right) Q^{n-m+i} P_{*}^{i},
$$

and for odd $p$,

$$
\begin{aligned}
P_{*}^{m} \beta Q^{n} & =\sum_{i}(-1)^{m+i}\left(\begin{array}{c}
(n-m)(p-1)-1 \\
m-i p
\end{array}\right) \beta Q^{n-m+i} P_{*}^{i} \\
& =+\sum_{i}(-1)^{m+i}\left(\begin{array}{c}
(n-m)(p-1)-1 \\
m-i p-1
\end{array}\right) Q^{n-m+i} P_{*}^{i} \beta
\end{aligned}
$$

They are easily derived from $(3 a)-(3 b)$ respectively: express the right hand sides of (3a)-(3b) as power series in $u, v$ and $\left(1-u^{-1} v\right)$ by (1), then express them as power series in $u$ and $v$ by the binomial theorem, and finally equate the coefficients of $u^{-m} v^{n}$.

7. Proof of the Adem relations $(2 a)-(2 d)$. This is based on May's proof $[4, \S 4]$; we shall show that $(2 a)-(2 d)$ are linear combinations of equations derived in that proof. First we recall some notation and the definition of the operations. Throughout this section $x$ is a homology class of degree $q$. If $p$ is odd, then we write

$$
m=\frac{1}{2}(p-1), \quad \nu(2 r)=(-1)^{r}, \quad \nu(2 r+1)=(-1)^{r} m !
$$

where $r$ is any integer. Modulo $p$ we have $(p-1) !=-1$ (Wilson's theorem), so

$$
(m !)^{2}=(-1)^{m+1} \text {. }
$$

For all $p$ and for $i \geqslant 0$, let $e_{i}$ be the standard generator of $H_{i}(\mathbf{Z} / p ; \mathbf{Z} / p) \cong \mathbf{Z} / p$ as in [4, 1.2]; for $i<0$, let $e_{i}$ be 0 . Let $\theta$ be the structure map inducing the operations and put

$$
D_{i} x=\theta_{*}\left(e_{i} \otimes x^{p}\right) \text {. }
$$

The definition $[4,2.2]$ of the operations then amounts to: for $p=2$,

$$
D_{i-q} x=P_{i} x
$$


and for odd $p$,

$$
D_{(2 i-q)(p-1)} x=(-1)^{i} \nu(q)^{-1} P_{i} x, \quad D_{(2 i-q)(p-1)-1} x=(-1)^{i} \nu(q)^{-1} \beta P_{i} x .
$$

Next we quote the necessary equations from $[4,4]$. They are equations $(e),(g)$ and $(i)$ of the proof of $[4,4.7]$, and say: for $p=2$,

$$
\sum_{k}\left(\begin{array}{c}
s-k \\
k
\end{array}\right) D_{r+2 k-s} D_{s-k} x=\sum_{l}\left(\begin{array}{c}
r-l \\
l
\end{array}\right) D_{s+2 l-r} D_{r-l} x
$$

for odd $p$ and for $\varepsilon=0$ or 1 ,

$$
\begin{aligned}
\sum_{k}(-1)^{k} \nu(2 s)\left(\begin{array}{c}
s-k(p-1) \\
k
\end{array}\right) D_{2 r+(2 k p-2 s)(p-1)-\varepsilon} D_{2 s-2 k(p-1)} x \\
=\sum_{l}(-1)^{l+a m} \nu(2 r)\left(\begin{array}{c}
r-l(p-1) \\
l
\end{array}\right) D_{2 s+(2 l p-2 r)(p-1)-\varepsilon} D_{2 r-2 l(p-1)} x
\end{aligned}
$$

and

$$
\begin{aligned}
\sum_{k}(-1)^{k+q m} \nu & (2 s-1)\left(\begin{array}{c}
s-k(p-1)-1 \\
k
\end{array}\right) D_{2 r+(2 k p-2 s+1)(p-1)-\varepsilon} D_{2 s-2 k(p-1)-1} x \\
= & (1-\varepsilon) \sum_{l}(-1)^{l+q m} \nu(2 r)\left(\begin{array}{c}
r-l(p-1) \\
l
\end{array}\right) D_{2 s+(2 l p-2 r)(p-1)-1} D_{2 r-2 l(p-1)} x \\
& -\sum_{l}(-1)^{l} \nu(2 r-1)\left(\begin{array}{c}
r-l(p-1)-1 \\
l
\end{array}\right) D_{2 s+(2 l p-2 r+1)(p-1)-\varepsilon} D_{2 r-2 l(p-1)-1} x .
\end{aligned}
$$

Finally we prove $(2 a)-(2 d)$ by forming linear combinations of $(11 a)-(11 c)$. First take $p=2$. Put $r=a-q, s=b-q$ in $(11 a)$, multiply both sides by $u^{a} v^{b}$, and sum over $a$ and $b$ to get

$$
\sum_{a, b, k}\left(\begin{array}{c}
b-k-q \\
k
\end{array}\right) u^{a} v^{b} D_{a-b+2 k} D_{b-k-q} x=\sum_{a, b, l}\left(\begin{array}{c}
a-l-q \\
l
\end{array}\right) u^{a} v^{b} D_{-a+b+2 l} D_{a-l-q} x .
$$

Change the summation variables on the left hand side to $i, j, k$, where $i=a+k+q$, $j=b-k$. The left hand side becomes

$$
\sum_{i, j, k}\left(\begin{array}{c}
j-q \\
k
\end{array}\right) u^{i-k-q} v^{j+k} D_{i-(q+j)} D_{j-q} x .
$$

By the binomial theorem and $(10 a)$, this is

$$
\sum_{i, j} u^{i-a} v^{j}\left(1+u^{-1} v\right)^{i-a} P_{i} P_{j} x
$$

(note that $\operatorname{deg} D_{i-q} x=\operatorname{deg} P_{j} x=q+j$ ). By (1) therefore (since we are working modulo 2), the left hand side of $(12)$ is $(u+v)^{-a} P(u) P(t) x$. Similarly the right hand side is $(u+v)^{-a} P(v) P(s) x$. So (12) implies $(2 a)$, for $p=2$, as required.

Next take $p$ to be odd. The calculations are similar, but more complicated. First put $r=a(p-1)-q m, s=b(p-1)-q m$ in $(11 b)$ and $(11 c)$, multiply both sides by $(-1)^{a+b} u^{a} v^{b}$, 
and sum over $a$ and $b$ to get equations analogous to (12). Transform the left hand sides as for $p=2$, using the change of variables $i=a+k+q m, j=b-k$, and eliminating the $\nu(d)$ by $(8 a)$. Transform the right hand sides in a similar manner. After using $(8 b)$ to eliminate $(m !)^{2}$ one obtains

$$
(-1)^{a}(v-u)^{-q m} \beta^{\varepsilon} P(u) P(t) x=(-1)^{q}(v-u)^{-q m} \beta^{\varepsilon} P(v) P(s) x
$$

and

$$
\begin{array}{r}
(-1)^{a}(v-u)^{-a m}\left(1-u^{-1} v\right)^{-1} \beta^{\varepsilon} P(u) \beta P(t) x \\
=(1-\varepsilon)(-1)^{a}(v-u)^{-a m} \beta P(v) P(s) x
\end{array}
$$

$$
-(-1)^{a}(v-u)^{-q m}\left(1-v^{-1} u\right)^{-1} \beta^{s} P(v) \beta P(s) x .
$$

Multiply these equations by $(-1)^{q}(v-u)^{a m}$ and $(-1)^{a}(v-u)^{a m}\left(1-u^{-1} v\right)$, respectively. The first equation gives (2a) for $\varepsilon=0$ and $(2 b)$ for $\varepsilon=1$, and the second gives $(2 c)$ for $\varepsilon=0$ and $(2 d)$ for $\varepsilon=1$. This completes the proof.

8. Proof of the Nishida relations $(5 a)-(5 b)$. The proof uses the formally similar Adem relations $(2 a)$ and $(2 c)$. To be precise, it uses the Adem relations for cohomology operations (Section 2, Remark 2) dualized and conjugated. Since the dual of the Bockstein is the Bockstein (up to sign) and the conjugate $c \beta$ of the Bockstein is $-\beta$, these relations are: for all $p$,

$$
c P_{*}\left(u^{-1}\right) c P_{*}\left(t^{-1}\right)=c P_{*}\left(v^{-1}\right) c P_{*}\left(s^{-1}\right),
$$

and for odd $p$,

$$
c P_{*}\left(u^{-1}\right) \beta c P_{*}\left(t^{-1}\right)=\left(1-u^{-1} v\right) \beta c P_{*}\left(v^{-1}\right) c P_{*}\left(s^{-1}\right)+u^{-1} v c P_{*}\left(v^{-1}\right) \beta c P_{*}\left(s^{-1}\right) .
$$

The idea of the proof is to express the $Q^{i}$ in terms of the $c P_{*}^{i}$, which is essentially done in $[4,9.1]$, and so reduce $(5 a)$ and $(5 b)$ to $(13 a)$ and $(13 b)$. To express the $Q^{i}$ in terms of the $c P_{*}^{i}$, we proceed as follows. Let $X$ be the $E_{\infty}$ space or $H_{\infty}$ ring spectrum we are considering. We have a composite map

$$
\theta d: B \mathbf{Z} / p \times X \rightarrow X
$$

where $B \mathbf{Z} / p$ is the classifying space of $\mathbf{Z} / p$ (so that $H_{*}(B \mathbf{Z} / p ; \mathbf{Z} / p)=H_{*}(\mathbf{Z} / p ; \mathbf{Z} / p)$ ), $\theta$ is the structure map defining the operations $Q^{i}=P_{i}$ by (9), (10a), (10b) and $d$ is the "diagonal" map whose effect in homology is described by [4, 9.1]. Let $x \in H_{s}(X ; \mathbf{Z} / p)$. From $[4,9.1]$ we obtain the equations: for $p=2$,

and for odd $p$,

$$
\theta_{*} d_{*}\left(e_{r} \otimes x\right)=\sum_{k} \theta_{*}\left(e_{r+2 k-s} \otimes\left(P_{*}^{k} x\right)^{2}\right),
$$

$$
\theta_{*} d_{*}\left(e_{2 r(p-1)} \otimes x\right)=\nu(s) \sum_{k}(-1)^{k} \theta_{*}\left(e_{(2 r+2 k p-s)(p-1)} \otimes\left(P_{*}^{k} x\right)^{p}\right) .
$$

Write

$$
e(z)=\left\{\begin{array}{lll}
\sum_{i} z^{i} e_{i} & \text { if } & p=2, \\
\sum_{i} z^{i} e_{2 i(p-1)} & \text { if } & p \text { is odd }
\end{array}\right.
$$


We now claim that, for all $p$,

$$
\theta_{*} d_{*}(e(-z) \otimes x)=Q(z) P_{*}\left(z^{-1}\right) x
$$

Indeed one can prove this by applying (15) to the left hand side, then $(14 a)-(14 b)$, then (9), then $(10 a)-(10 b)$ (note that $P_{i}$ is $Q^{i}$ in the present application, and that $\operatorname{deg} P_{*}^{i} x$ is $s-i$ if $p=2$ or $s-2 i(p-1)$ if $p$ is odd), and finally (8a). Since $c P_{*}\left(z^{-1}\right)=P_{*}\left(z^{-1}\right)^{-1}$ (by (4)), (16) gives: for all $p$

$$
Q(z) x=\theta_{*} d_{*}\left(e(-z) \otimes c P_{*}\left(z^{-1}\right) x\right) .
$$

Equation (17) expresses the $Q^{i}$ in terms of the $c P_{*}^{i}$. To proceed further, we need the values of the Steenrod operations in $H_{*}(\mathbf{Z} / p ; \mathbf{Z} / p)$. These are well-known: from [4, proof of 4.6, equations $(c)$ and $(d)]$ and from $[4,1.2(2)]$ we find: for $p=2$,

$$
P_{*}^{k} e_{r}=\left(\begin{array}{c}
r-k \\
k
\end{array}\right) e_{r-k}
$$

and for odd $p$,

$$
\begin{array}{r}
\beta e_{2 r(p-1)}=e_{2 r(p-1)-1}, \\
P_{*}^{k} e_{2 r(p-1)}=\left(\begin{array}{c}
(r-k)(p-1) \\
k
\end{array}\right) e_{2(r-k)(p-1)}, \\
P_{*}^{k} e_{2 r(p-1)-1}=\left(\begin{array}{c}
(r-k)(p-1)-1 \\
k
\end{array}\right) e_{2(r-k)(p-1)-1} .
\end{array}
$$

With $e(z)$ as in (15), these equations combined with the binomial theorem give: for all $p$,

and for odd $p$,

$$
P_{*}\left(u^{-1}\right) e(-v)=e(-t)
$$

$$
P_{*}\left(u^{-1}\right) \beta e(-v)=\left(1-u^{-1} v\right)^{-1} \beta e(-t),
$$

where $t=v\left(1-u^{-1} v\right)^{p-1}$ as in (1). Inverting $P_{*}\left(u^{-1}\right)$ by (4) gives: for all $p$,

and for odd $p$,

$$
c P_{*}\left(u^{-1}\right) e(-t)=e(-v) \text {, }
$$

$$
c P_{*}\left(u^{-1}\right) \beta e(-t)=\left(1-u^{-1} v\right) \beta e(-v) .
$$

We can now prove $(5 a)$. For all $p,(17)$ gives

$$
c P_{*}\left(u^{-1}\right) Q(t) x=c P_{*}\left(u^{-1}\right) \theta_{*} d_{*}\left(e(-t) \otimes c P_{*}\left(t^{-1}\right) x\right) .
$$

But $\theta_{*} d_{*}$ commutes with $c P_{*}\left(u^{-1}\right)$, since it is induced by a map $\theta d$, so the right hand side is

$$
\theta_{*} d_{*} c P_{*}\left(u^{-1}\right)\left(e(-t) \otimes c P_{*}\left(t^{-1}\right) x\right)
$$

Apply the Cartan formula (6), then (18a) and the Adem relation (13a), to get

$$
\theta_{*} d_{*}\left(e(-v) \otimes c P_{*}\left(v^{-1}\right) c P_{*}\left(s^{-1}\right) x\right) \text {. }
$$

By (17) this is $Q(v) c P_{*}\left(s^{-1}\right) x$, as required. This completes the proof of $(5 a)$. 
Similarly we can prove $(5 b)$. Here $p$ is odd. By (17)

$$
c P_{*}\left(u^{-1}\right) \beta Q(t) x=c P_{*}\left(u^{-1}\right) \beta \theta_{*} d_{*}\left(e(-t) \otimes c P_{*}\left(t^{-1}\right) x\right) .
$$

Now $\beta$ commutes with $\theta_{*} d_{*}$ just as $c P_{*}\left(u^{-1}\right)$ does, and $\beta$ is a derivation, so the right hand side is

$$
\theta_{*} d_{*} c P_{*}\left(u^{-1}\right)\left(\beta e(-t) \otimes c P_{*}\left(t^{-1}\right) x+e(-t) \otimes \beta c P_{*}\left(t^{-1}\right) x\right)
$$

Apply (6), then (18a)-(18b) and (13a)-(13b), to get

$$
\begin{aligned}
\left(1-u^{-1} v\right) \theta_{*} d_{*}\left(\beta e(-v) \otimes c P_{*}\left(v^{-1}\right) c P_{*}\left(s^{-1}\right) x\right. & \left.+e(-v) \otimes \beta c P_{*}\left(v^{-1}\right) c P_{*}\left(s^{-1}\right) x\right) \\
& +u^{-1} v \theta_{*} d_{*}\left(e(-v) \otimes c P_{*}\left(v^{-1}\right) \beta c P_{*}\left(s^{-1}\right) x\right) .
\end{aligned}
$$

Since $\beta$ is a derivation commuting with $\theta_{*} d_{*}$, this is

$$
\left(1-u^{-1} v\right) \beta \theta_{*} d_{*}\left(e(-v) \otimes c P_{*}\left(v^{-1}\right) c P_{*}\left(s^{-1}\right) x\right)+u^{-1} v \theta_{*} d_{*}\left(e(-v) \otimes c P_{*}\left(v^{-1}\right) \beta c P_{*}\left(s^{-1}\right) x\right),
$$

and by (17) this is

$$
\left(1-u^{-1} v\right) \beta Q(v) c P_{*}\left(s^{-1}\right) x+u^{-1} v Q(v) \beta c P_{*}\left(s^{-1}\right) x
$$

as required. This completes the proof.

\section{REFERENCES}

1. S. R. Bullett and I. G. Macdonald, On the Adem relations, Topology 21 (1982), 329-332.

2. F. R. Cohen, T. J. Lada and J. P. May, The homology of iterated loop spaces, Lecture Notes in Mathematics 533 (Springer-Verlag, 1976). 35-88.

3. E. Dyer and R. K. Lashof, Homology of iterated loop spaces, Amer. J. Math. 84 (1962),

4. J. P. May, A general algebraic approach to Steenrod operations, in F. P. Peterson, ed., The Steenrod algebra and its applications, Lecture Notes in Mathematics 168 (Springer-Verlag, 1970), 153-231.

5. J. P. May, $H_{\infty}$ ring spectra and their applications, in R. J. Milgram, ed., Algebraic and geometric topology, Proc. Symp. Pure Math. 32 (1978), part 2, 229-243. 104-109.

6. G. Nishida, Cohomology operations in iterated loop spaces, Proc. Japan Acad. 44 (1968),

Department of Mathematics

UNIVERSITY OF GLASGOW

GLASGOW G12 8QW 